Nervenarzt 2009 $\cdot 80: 1506$

DOI 10.1007/s00115-009-2891-1

(c) Springer-Verlag 2009

W. Hacke ${ }^{1} \cdot$ H.C. Diener ${ }^{2} \cdot$ H.P. Hartung ${ }^{3} \cdot$ C. Elger ${ }^{4} \cdot$ T.H. Brandt ${ }^{5}$

${ }^{1}$ Neurologische Klinik, Universitätsklinikum Heidelberg

${ }^{2}$ Klinik und Poliklinik für Neurologie, Universitätsklinikum Essen

${ }^{3}$ Neurologische Klinik, Universitätsklinikum Düsseldorf

${ }^{4}$ Klinik für Epileptologie, Universitätsklinikum Bonn

${ }^{5}$ Institut für Klinische Neurowissenschaften, Klinikum

Großhadern, Universität München

\title{
Erratum zu: Messung von Publikationsleistungen
}

Hacke W, Diener HC, Hartung HP et al (2009)

Messung von Publikationsleistungen. Nervenarzt 80:1226-1238

In $\bullet$ Tab. 1 des genannten Beitrags wurden die Daten zum Thema Parkinson leider nicht berücksichtigt. Bitte entnehmen Sie die Informationen zum Themenkomplex Parkinson der hier dargestellten Tabellenergänzung. Wir bitten um entsprechende Beachtung.

\author{
Korrespondenzadresse \\ Prof. Dr. W. Hacke \\ Neurologische Klinik, \\ Universitätsklinikum Heidelberg \\ Im Neuenheimer Feld 400, 69120 Heidelberg \\ werner_hacke@med.uni-heidelberg.de
}

Die Online-Version des Originalartikels können Sie unter http://dx.doi.org/10.1007/s00115-0092836-8 finden.

\section{Tab. 1 Autoren, Institut und Länderranking (Quelle: ISI Web of Science, Stand Januar 2009)}

\begin{tabular}{|c|c|c|c|c|c|c|}
\hline Thema & $\begin{array}{l}\text { Gesamtzahl Artikel } \\
\text { 1987-2008 }\end{array}$ & $\begin{array}{l}\text { Top Autoren } \\
\text { weltweit }\end{array}$ & $\begin{array}{l}\text { Beste Positionen deutscher } \\
\text { Autoren innerhalb Top } 100 \\
\text { (max. 5) }\end{array}$ & $\begin{array}{l}\text { Top } 5 \text { Institutionen } \\
\text { weltweit }\end{array}$ & $\begin{array}{l}\text { Beste Positionen } \\
\text { deutscher Zentren } \\
\text { (max. 5) }\end{array}$ & $\begin{array}{l}\text { Länderranking mit } \\
\text { Zahl der Artikel } \\
\text { (gerundet) }\end{array}$ \\
\hline Parkinson & 29.251 & $\begin{array}{l}\text { Agid } \\
\text { Lees } \\
\text { Jenner } \\
\text { Jankovic } \\
\text { Lang }\end{array}$ & $\begin{array}{l}7 \text { Oertel } \\
9 \text { Poewe } \\
10 \text { Mueller } \\
13 \text { Riederer } \\
18 \text { Gasser }\end{array}$ & $\begin{array}{l}\text { Harvard } \\
\text { Salpetriere } \\
\text { Inst N London } \\
\text { Columbia } \\
\text { Penn U }\end{array}$ & $\begin{array}{l}15 \text { Tübingen } \\
16 \text { München } \\
22 \text { Würzburg } \\
24 \text { Bochum } \\
31 \text { Marburg }\end{array}$ & $\begin{array}{l}\text { USA } 13.900 \\
\text { GER } 3.700 \\
\text { GB } 3.700 \\
\text { JAP } 2.900 \\
\text { ITA } 2.500\end{array}$ \\
\hline
\end{tabular}

